Document downloaded from:

http://hdl.handle.net/10251/60641

This paper must be cited as:

Romero García, V.; Fuster García, E.; Sánchez Pérez, JV.; García Raffi, LM.; Blasco, X.; Herrero Durá, JM.; Sanchís Saez, J. (2007). Genetic Algorithm in the Optimization of the Acoustic Attenuation System. Lecture Notes in Computer Science. 4507:614-621. doi:10.1007/978-3-540-73007-1_74.

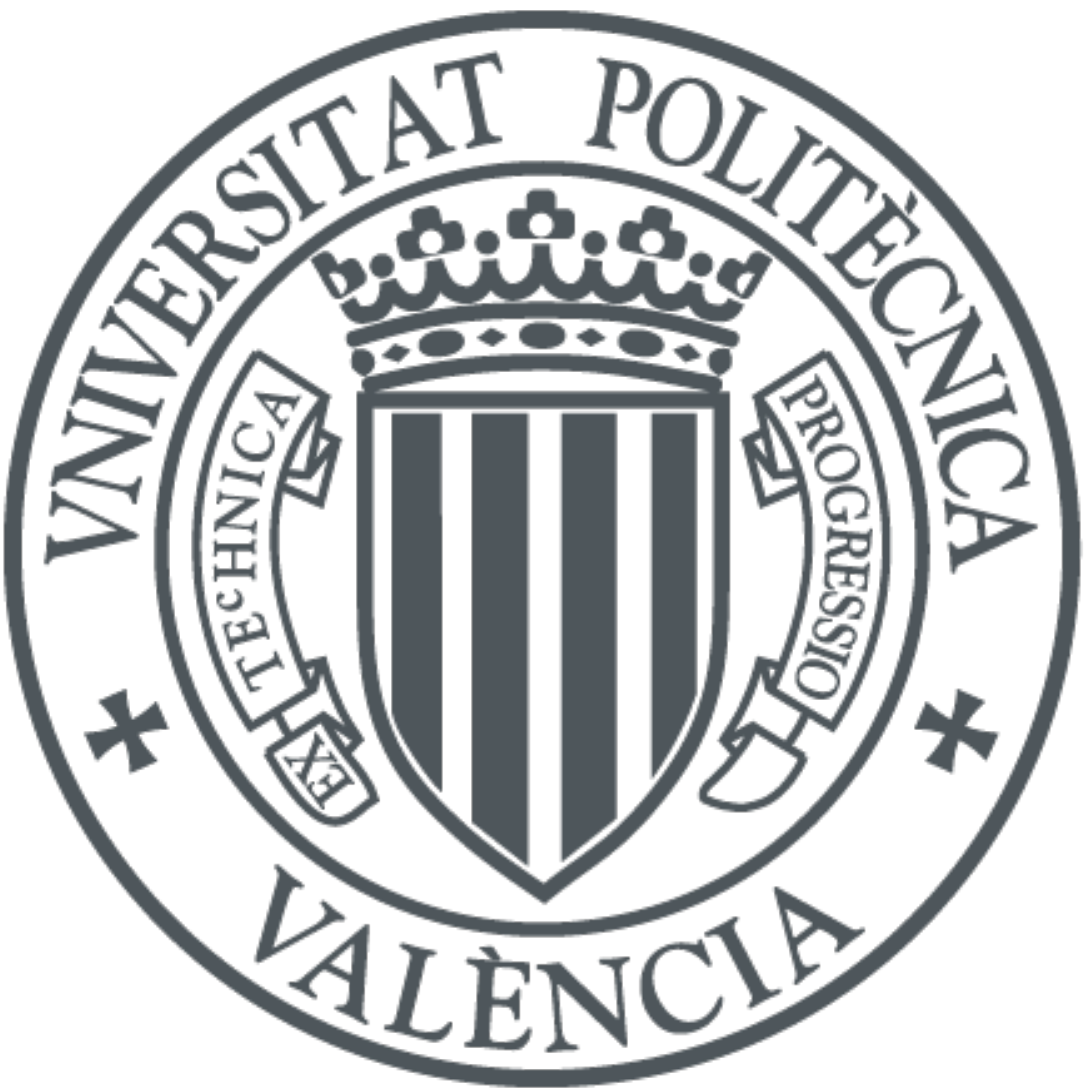

The final publication is available at

http://dx.doi.org/10.1007/978-3-540-73007-1_74

Copyright Springer Verlag (Germany)

Additional Information 


\title{
Genetic Algorithm in the Optimization of the Acoustic Attenuation Systems
}

\author{
V. Romero-García ${ }^{1}$, E. Fuster-Garcia ${ }^{1}$, J.V. Sánchez-Pérez ${ }^{1}$, \\ L.M. Garcia-Raffi ${ }^{2}$, X. Blasco ${ }^{3}$, J.M. Herrero ${ }^{3}$, and J. Sanchis ${ }^{3}$ \\ ${ }^{1}$ Grupo de Acústica Arquitectónica y del Medioambiente (ACARMA) \\ Department of Applied Physics \\ jusanc@fis.upv.es \\ ${ }^{2}$ Instituto de Matemática Pura y Aplicada \\ Department of Applied Mathematics \\ Imgarcia@mat .upv.es \\ ${ }^{3}$ Predictive Control and Heuristic Optimization Group \\ Department of Systems Engineering and Control \\ Polytechnic University of Valencia \\ xblasco@isa.upv.es \\ http://ctl-predictivo.upv.es
}

\begin{abstract}
It is well known that Genetic Algorithms (GA) is an optimization method which can be used in problems where the traditional optimization techniques are difficult to be applied. Sonic Crystals (SC) are periodic structures that present ranges of sound frequencies related with the periodicity of the structure, where the sound propagation is forbidden. This means that in the acoustic spectrum there are ranges of frequencies with high acoustic attenuation. This attenuation can be improved producing vacancies in the structure. In this paper we use a parallel implementation of a GA to optimize those structures, by means of the creation of vacancies in a starting $\mathrm{SC}$, in order to obtain the best acoustic attenuation in a predetermined range of frequencies. The cost function used in GA is based on the Multiple Scattering Theory (MST), which is a self consistent method for calculating acoustic pressure in SCs. As a final result we achieve a quasi ordered structures that presents a high acoustic attenuation in a predetermined range of frequencies, independent of the periodicity of the SC.
\end{abstract}

\section{Introduction}

A Genetic Algorithm (GA) is a global search method based on a simile of the natural evolution. GAs have demonstrated good performance for difficult problems where the function to minimize is complicated. In this work we applied this optimization method to improve the acoustical properties of the Sonic Crystal (SC) [1] [2], a kind of structures used in acoustics.

In the last few years the propagation of the acoustic waves in heterogeneous materials whose acoustic properties vary periodically in space have attracted considerable interest. The so-called SCs are the typical example of this kind of 
materials in the range of the acoustic frequencies. These systems are defined as periodic structures with strong modulation of the elastic constants between the scatterers and the surrounding material.

Recently, the strategy to enhance SC properties has been based on the use of scatterers with acoustical properties added. The use of local resonators [3] or Helmholtz resonators [4] as scatterers have produced very good results Some authors also have built new structures with scatterers made up of porous material improving the attenuation capability of the SCs [5]. However, the use of SCs as outdoor acoustic barriers requires scatterers made up of robust and long-lasting materials. This is the reason why it seems interesting to analyze the possibility of optimizing the attenuation capability of SC made with rigid scatterers like wood, $\mathrm{PVC}$ or aluminium. The creation of vacancies in a $\mathrm{SC}$ improves the attenuation capability of the SC [6]. However, it does not exist any generic rule about the creation of vacancies in a SC. In fact, similar structures can produce very different acoustic fields behind of them.

Because of the complexity of mathematical functions involved in SC calculus, GA turns up as a tool specially indicated for this kind of problems [7] [8]. This procedure can work together with the Multiple Scattering theory (MST) which is a self-consistent method for calculating the acoustic pressure including all orders of scattering [9]. Given a starting SC, the GA generates quasi ordered structures (QOS) offspring by means of the creation of vacancies that are classified in terms of a cost function based on the pressure values at a specific point. The sound scattered pressure by every structure analyzed by GA is performed by a twodimensional (2D) MST. In the present work, it is shown an improvement of the Genetic Algorithm based on Parallel implementation and as a consequence, new and better results are obtained to design QOSs made with rigid cylinders that attenuate sound in a predetermined band of frequencies.

\section{Sonic Crystals (SCs)}

SCs are arrays of scatterers placed periodically in space whose physical properties are different to the surrounding material. In the low frequency range, SCs behave as an homogeneous medium with an acoustic impedance greater than that of the air. Then SCs can work as refractive devices. Moreover, SCs present band gaps, i.e., ranges of sound frequencies where the sound propagation inside the crystal is forbidden. The presence of these band gaps is explained by the well-known Bragg's law. The reflections inside the crystal, and consequently the position of the gaps depend on the lattice constant, i.e., on the geometry of the SC. The existence, in periodic media, of an absolute band gap where the propagation of sound is forbidden for every incidence direction, can have a profound impact on several scientific and technological disciplines, for example, in the design of acoustic filters or acoustic barriers.

Some studies have showed that there are three important parameters for the spectral gap creation [11]. One is the density ratio $y=\frac{\rho_{s}}{\rho_{h}}$ between the scattering material and the host material densities. The second one is the filling factor, $f f=\frac{V_{s}}{V}$, that shows the volume occupied by the scattering material respect to 
the total volume. The last parameter is the topology used to design the SC. It was demonstrated that the density ratio plays an important role in the gap creation: SCs built with scatterers of high density embedded in a host material of low density are better to create the spectral gap than another kind of configurations. Moreover the optimum value of the filling factor, $f f$, to the gap creation has been ranged between $10 \%$ and $50 \%$.

In this work we use a SC built by aluminium cylinders of $2 \mathrm{~cm}$ of radius as scatterers embedded in air (Network topology). Due to the fact that those structures present a high density ratio, and the maximum filling factor is $f f=$ 0.36 , we ensure that our structure is well designed to the gap creation. Now we want to find the best filling factor and space distribution of scatterers that present the best acoustical properties. GA together with the MST is a good procedure to achieve our objective.

\section{Cost Function and Chromosome Description}

The mechanism used by GA in this work is the creation of vacancies in the starting SC. Fig. 1 shows the starting SC and a QOS offspring generated by GA by means of the creation of vacancies. Using this procedure we can vary the filling factor and, at the same time, evaluate different spaces of configuration. Each QOS will be considered as an individual. The chromosome that represents each QOS, is a real vector with values in $[0,1]$ range. Each coordinate represents the existence or not of a cylinder at a specific position of the scatterer (beginning with the cylinder a the left top corner of the SC and following by columns until right bottom corner, see starting $\mathrm{SC}$ at figure 1$)$. Values in $[0,0.5]$ means there is a vacancy, in opposition values in $[0.5,1]$ means there is a cylinder.

In this work we are interested in maximizing the sound attenuation for a predetermined range of frequencies not dependent on the lattice constant, at a point located behind the crystal.

The acoustic attenuation in a point $(x, y)$ and for a incidence frequency $\nu$ is:

$$
\operatorname{Attenuation}(d B)=20 \log \frac{\left|P_{\text {direct }}(x, y)\right|}{\left|P_{\text {interfered }}\left(x, y, X_{c y l}, Y_{c y l}, \nu, r_{l}\right)\right|}
$$

where the interfered pressure is determined by the MST. This pressure depends on the position and on the radius of the scatterers and the incidence frequency. In the equation (1) we can see that for a point $(x, y)$, a value of incidence frequency $\nu$ and a value of cylinder radius $r_{l}$, it is possible to find a configuration of cylinders that minimize the $P_{\text {interferred }}$, that means, maximize the acoustic attenuation. If we are interested in maximizing the sound attenuation in a predetermined range of frequencies at a point of coordinates $(x, y)$ we have to define a new function that we have to minimize in order to achieve the maximum acoustic attenuation. To do that, we define our cost function based on the MST

$$
J(\mathbf{x})=\bar{p}+\sqrt{\frac{\sum_{j=1}^{N}\left(\bar{p}-\left|p_{j}(\mathbf{x})\right|\right)^{2}}{N^{2}}}
$$




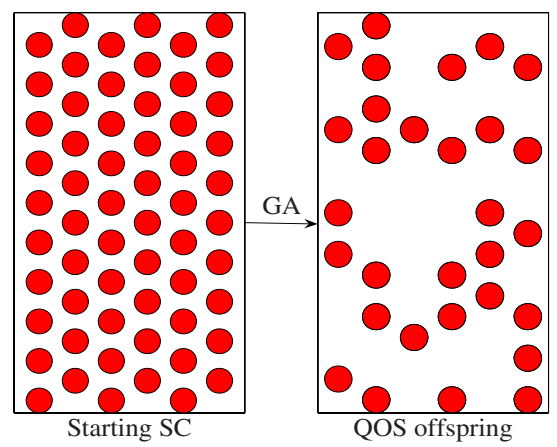

Fig. 1. Starting SC and a possible QOS offspring

where

$$
\bar{p}=\sum_{j=1}^{N} \frac{\left|p_{j}(\mathbf{x})\right|}{N}
$$

represents the mean pressure in the range of frequencies $\left[\nu_{1}, \nu_{N}\right]$ and $N$ represents the number of frequencies considered in this range. In our case, we use $N=13$. The second term in equation (2) represents the mean deviation. The variable under study is $\mathbf{x}=\left(X_{c y l}, Y_{c y l}\right)$ a vector that contains the information about the space configuration of the QOSs.

\section{Parallel Genetic Algorithm}

A GA is an optimization technique that looks for the solution of the optimization problem, imitating species evolutionary mechanism [12].

In an optimization problem, there is a function to optimize (cost function) and a zone where to look for (search space). Every point of the search space had an associated value of the function.

The different points of the search space are the different individuals of population. Similarly to natural genetic, every different individual is characterized by a chromosome and in the optimization problem, this chromosome is made by the point coordinates in the search space. The cost function value for an individual has to be understood as the adaptation level to the environment for such individual.

Evolutionary mechanism, that is, the rules for changing populations throughout generations is performed by Genetic Operators. A general GA evolution mechanism could be described as follows:

From an initial population (randomly generated), the next generation is obtained as:

1. Some individuals are selected for the next generation. This selection is made depending on adaptation level (cost function value). Such individuals with better $J(\mathbf{x})$ value have more possibilities to be selected. 
2. To explore search space, an exchange of information between individuals is performed by crossover. That produces a gene exchange between chromosomes. The rate of individuals to crossover is fixed by $P_{c}$, crossover probability.

3. An additional search space exploration is performed by mutation. Some individuals are subject to a random variation in their genes. The rate of individuals to be mutated is set by mutation probability $P_{m}$.

In this general framework, there are several variation in the GA implementation; different gene codification, different genetic operator implementation, etc. Implementation for the present work has the following characteristics:

1. Real value codification, each gene has a real value, the interpretation of the chromosome has been detailed in previous section.

2. $J(\mathbf{x})$ is not directly used as cost function. A linear 'ranking' operation is performed [13]. Ranking operation prevents the algorithm from exhausting, it avoids clearly dominant individuals prevailing too soon.

3. Selection is made by the operator known as Stochastic Universal Sampling (SUS) [14].

4. For crossover it is used intermediate recombination operator [15]. Chromosomes sons $\left(\mathbf{x}_{1}^{\prime}\right.$ and $\left.\mathbf{x}_{2}^{\prime}\right)$ are obtained through following operation on chromosomes fathers $\left(\mathbf{x}_{1}\right.$ and $\left.\mathbf{x}_{2}\right)$ :

$$
\mathbf{x}_{1}^{\prime}=\alpha_{1} \cdot \mathbf{x}_{1}+\left(1-\alpha_{1}\right) \mathbf{x}_{2} ; \mathbf{x}_{2}^{\prime}=\alpha_{2} \cdot \mathbf{x}_{2}+\left(1-\alpha_{2}\right) \mathbf{x}_{1} ; \alpha_{1}, \alpha_{2} \in[-d, 1+d]
$$

$\alpha_{1}$ and $\alpha_{2}$ have to be generated for each gene increasing search capabilities but with a higher computational cost.

Implemented GA has been adjusted as follows: $\alpha_{1}=\alpha_{2}$ and generated for each chromosome, $d=0$ and $P_{c}=0.8$.

5. Mutation operation is done with a probability $P_{m}=0.1$ and a normal distribution with standard deviation set to $20 \%$ of search space range.

The high computational cost of SC optimization problem produces huge execution time, i.e. in a standard execution (population of 360 individuals, 250 generations) time is around 104 hours. Improvements of execution time have been obtained with a parallel implementation of the GA described. Several alternative for parallelization are possible [16] the selected one is the configuration Master-Slave. For this architecture there is one processor working as Master, executing tasks of the GA (ranking, selection, crossover and mutation), and the rest evaluate fitness function of a subpopulation (see Fig. 2).

The Master has to send subpopulation to each Slave, who makes fitness evaluation and returns results to the Master. The Master works in a synchronous way, waiting for all fitness value from all Slaves. After receiving all fitness values the Master performs the evolution to produce the next generation (genetic operators are executed) and sends to the Slaves the new population for fitness evaluation. This type of implementation is the most simple and does not change GA operators and behaviour. The time reduction is significative since the overall time is 


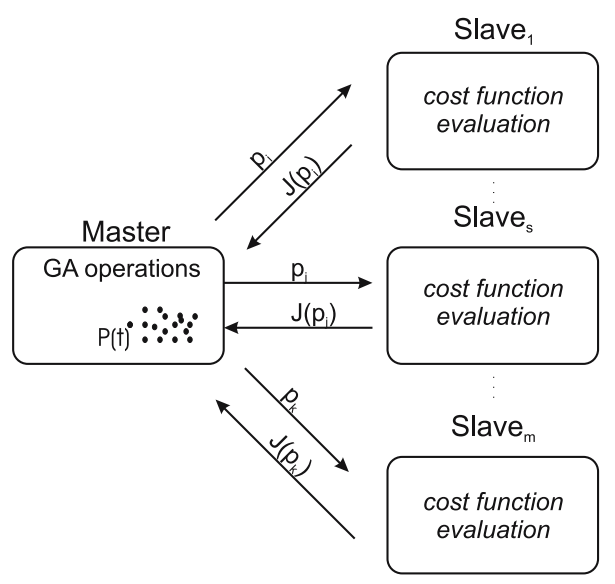

Fig. 2. Master/Slave architecture for Parallel Genetic Algorithm

divided by the number of Slaves. For the problem proposed, with 5 Slaves, the total execution is reduced to 21 hours.

All developments (GA and SC models) have been made in Matlab ${ }^{\circledR}$, parallelization has been done using Matlab Distributed Computing Toolbox and Matlab Distributed Computing Engine.

\section{$5 \quad$ Results}

In this point we present some of our main results. In this work we have analyzed width ranges of $600 \mathrm{~Hz}$ centered at several frequencies $(800,1100,1300,1700$, 2000, 2300, $3090 \mathrm{~Hz}$ ) above the first Bragg's peak. On the Fig. 3 we present the results corresponding to the ranges of frequencies centered at 1700 and $3090 \mathrm{~Hz}$ respectively. On the left hand of the Fig. 3 we present the schemes of cylinders of the QOSs generated by the design tool described above. On the right hand the acoustic attenuation spectra calculated by the MST for the starting SC (continuous line) and for the optimized QOSs (dashed line) is shown.

The creation of attenuation peaks in ranges of frequencies independents on the geometry of the starting SC using rigid scatterers has been the goal of this paper. As one can see on the Fig. 3, the peak attenuation in the spectra of the optimized QOS appears in the chosen frequency range, and this peak is absent in the spectra of the starting SC. Notice that the acoustic attenuation level in the frequency range in the starting $\mathrm{SC}$ is much lower than the QOS one. Even in some case the starting SC produces sound reinforcement. Moreover, the total number of cylinders in the optimized QOSs is also lower than the starting SC one. In our results the number of cylinders is ranged between $36.7 \%$ and $60 \%$. 

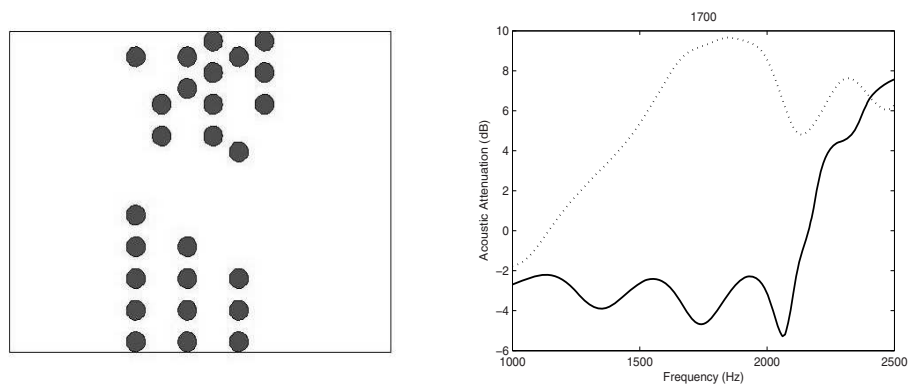

(a)
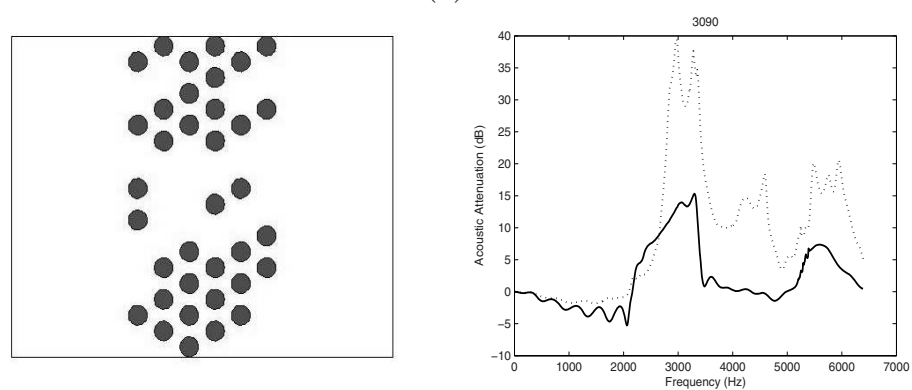

(b)

Fig. 3. Optimized QOSs and its spectrum. On the left hand the plot presents the schemes of cylinders of the optimized QOSs. On the right hand the plots show the acoustic attenuation spectra calculated by the MST for the starting SC (continuous line) and for the optimized QOSs (dashed line). (a) Optimization corresponding to the central frequency of $1700 \mathrm{~Hz}$. (b) Optimization corresponding to the central frequency of $3000 \mathrm{~Hz}$.

These results constitute a useful tool to design acoustic barriers based on $\mathrm{SC}$ with no need for sophisticated scatterers. The technological advantages of using QOS with rigid cylinders as scatterers are: high resistance for use outdoors, constructive simplicity and low cost due to the reduction in volume of the crystal.

\section{Conclusions}

This work shows an important and successful application of a Genetic Algorithm with a parallel implementation. SCs open the way for innovative application in noise reduction in several interesting areas as acoustic noise barriers for traffic or general devices for controlling the noise. The GA demonstrates an adequate optimization for a so complex problem and with the parallel implementation execution times are drastically reduced. Moreover, this method offers the possibility to test a wide range of $\mathrm{SC}$ adjustment in a reasonable time. 


\section{Acknowledgments}

The authors acknowledge financial support provided by the Spanish MEC (Project No. MAT2006-03097) and by the Generalitat Valenciana (Spain) under Grant No. GV/2007/191. This work also has been partially supported by MEC (Spanish government) and FEDER funds: projects DPI2005-07835, DPI20048383-C03-02 and GVA-026.

\section{References}

1. Martínez-Sala, R., Sancho, J., Sánchez Pérez, J.V., Llinares, J., Meseguer, F.: Sound atteuntaion by sculpture. Nature (London) 387, 241 (1995)

2. Hushwaha, M.S., Halevi, P., Martínez, G., Dobrynski, L., Djafari-Rouhani, B.: Theory of acoustic band structure of periodic elastic composites. Phys. Rev. B 49(4), 2313-2322 (1994)

3. Liu, Z., Zhang, X., Mao, Y., Zhu, Y.Y., Yang, Z., Xhan, C.T., Sheng, P.: Locally resonatn sonic materials. Science 289, 1734 (2000)

4. Hu, X., Chan, C.T., Zi, J.: Two dimensional sonic crystals with Helmholtz resonators. Phys. Rev. E 71, 055601 (2005)

5. Umnova, O., Attenborough, K., Linton, C.M.: Effects of porous covering on sound attenuation by poriodi arrays of cylinders. J. Acoust. Soc. Am 119, 278 (2006)

6. Caballero, D., Sánchez-Dehesa, J., Martínez-Sala, R., Rubio, C., Sánchez Pérez, J.V.S., Sanchis, L., Meseguer, F.: Suzuki phase in two-dimensional sonic crystals. Phys. Rev. B 64, 064303 (2001)

7. Hakansson, A., Sánchez-Dehesa, J., Sanchis, L.: Acoustic lens design by genetic algorithms. Phys. Rev. B 70, 214302 (2004)

8. Romero-García, V., Fuster, E., García-Raffi, L.M., Sánchez-Pérez, E.A., Sopena, M., Llinares, J., Sánchez-Pérez, J.V.: Band gap creation using quasiordered strutures based on sonic crystals. Appl. Phys. Lett. 88, 174104-1 174104-3 (2006)

9. Chen, Y.Y., Ye, Z.: Theoretical analysis of acoustic stop bands in two-dimensional periodic scattering arrays. Phys. Rev. E 64, 036616 (2001)

10. Economou, E.N., Sigalas, M.M.: Classical wave propagation in periodic structures: Cermet versus network topology. Phys. Rev. B 48(18), 13434 (1993)

11. Sigalas, M.M., Economou, E.N., Kafesaki, M.: Spectral gaps for electromagnietic and scalar waves: Possible explanation for certain differences. Phys. Rev. B 50(5), 3393 (1994)

12. Goldberg, D.E.: Genetic Algorithms in search, optimization and machine learning. Addison-Wesley, London (1989)

13. Bäck, T.: Evolutionaty Algorithms in theory and practice. Oxford University Press, New York (1996)

14. Baker, J.E.: Reducing bias and inefficiency in the selection algorithm. In: Proc. Second International Conference on Genetic Algorithms (1987)

15. Mühlenbein, H., Schlierkamp-Voosen, D.: Predictive Models for the Breeder Genetic Algorithm I. Continuous Parameter Optimization. Evolutionary Computation, vol. 1(1) (1993)

16. Cantú-Paz, E.: A summary of resaearch on parallel genetic algorithms. Technical Report 95007, Illinois Genetic Algorithms Laboratory. IlliGAL (1995) 